Bond University

Research Repository

\title{
Alexithymia in alcohol dependent patients is partially mediated by alcohol expectancy
}

Thorberg, Fred Arne; Young, Ross McD; Sullivan, Karen A.; Lyvers, Michael; Hurst, Cameron P.; Connor, Jason P.; Feeney, Gerald F X

Published in:

Drug and Alcohol Dependence

DOI:

10.1016/j.drugalcdep.2010.11.015

Licence:

CC BY-NC-ND

Link to output in Bond University research repository.

Recommended citation(APA):

Thorberg, F. A., Young, R. M., Sullivan, K. A., Lyvers, M., Hurst, C. P., Connor, J. P., \& Feeney, G. F. X. (2011). Alexithymia in alcohol dependent patients is partially mediated by alcohol expectancy. Drug and Alcohol Dependence, 116(1-3), 238-241. https://doi.org/10.1016/j.drugalcdep.2010.11.015

\section{General rights}

Copyright and moral rights for the publications made accessible in the public portal are retained by the authors and/or other copyright owners and it is a condition of accessing publications that users recognise and abide by the legal requirements associated with these rights.

For more information, or if you believe that this document breaches copyright, please contact the Bond University research repository coordinator. 
Alexithymia in alcohol dependent patients is partially mediated by alcohol expectancy

$$
\begin{aligned}
& \text { Fred Arne Thorberg, Ph.D. }{ }^{a, f} \\
& \text { Ross McD. Young, Ph.D. }{ }^{\text {a,e }} \\
& \text { Karen A. Sullivan, Ph.D. }{ }^{\text {a,b }} \\
& \text { Michael Lyvers, Ph.D. }{ }^{\text {c }} \\
& \text { Cameron P. Hurst, Ph.D. }{ }^{d} \\
& \text { Jason P. Connor, Ph.D. }{ }^{\text {e,f,g }}
\end{aligned}
$$

Gerald F. X. Feeney, MB, BCh. FRACP., ${ }^{\mathrm{e}, \mathrm{f}}$

Address correspondence to:

Fred Arne Thorberg

${ }^{\text {a }}$ Institute of Health and Biomedical Innovation, Queensland University of Technology 60 Musk Avenue, Kelvin Grove, Queensland 4059 Australia

$$
\text { telephone: (61) } 731386131
$$$$
\text { facsimile: (61) } 731386039
$$

e-mail: f.thorberg@qut.edu.au

${ }^{\mathrm{b}}$ School of Psychology and Counseling, Queensland University of Technology, Brisbane, Queensland, Australia

${ }^{\mathrm{c}}$ Department of Psychology, Bond University, Gold Coast, Queensland, Australia

d School of Public Health, Queensland University of Technology, Queensland, Brisbane, Australia

e Alcohol \& Drug Assessment Unit, Princess Alexandra Hospital, Brisbane, Queensland, Australia

${ }^{\mathrm{f}}$ Centre for Youth Substance Abuse Research, Faculty of Health, The University of Queensland, Brisbane, Queensland, Australia

${ }^{g}$ Discipline of Psychiatry, The University of Queensland, Brisbane, Queensland, Australia 


\begin{abstract}
Background: Up to fifty percent of alcohol dependent individuals have alexithymia, a personality trait characterised by difficulties identifying and describing feelings, a lack of imagination and an externalised cognitive style. Although studies have examined alexithymia in relation to alcohol dependence, no research exists on mechanisms underlying this relationship. The present study examined the mediational effect of alcohol expectancies on alexithymia and alcohol dependence. Methods: 230 outpatients completed the Toronto Alexithymia Scale (TAS-20), the Drinking Expectancy Questionnaire (DEQ) and the Alcohol Use Disorder Identification Test (AUDIT). Results: Regression analysis showed that alexithymia and alcohol dependence was, in two of three cases, partially mediated through alcohol expectancy. Conclusions: Alcohol expectancies of assertion and affective change show promise as mediators of alcohol dependence in individuals with alexithymia.
\end{abstract}

Keywords: Alexithymia, alcohol expectancies, alcohol dependence 
Alexithymia in alcohol dependent patients

\section{Introduction}

Alexithymia is a personality construct characterized by difficulties identifying feelings (DIF), difficulties describing feelings (DDF), a lack of imagination and an externally oriented cognitive style (EOT) (Nemiah et al., 1976). The prevalence of alexithymia in alcohol dependent populations is between 45-67\% (Thorberg et al., 2009). Previous research has identified significant positive relationships between alexithymia, heavy drinking and alcohol dependence (Thorberg et al., 2009; Thorberg et al., 2010). Furthermore, individuals with alexithymia and alcohol dependence have a younger age of onset, higher levels of dependence severity and stronger suicidal ideation (Sakuraba et al., 2005; Uzun et al., 2003). This suggests that those with alexithymia may be a more severe group of alcohol dependent individuals than their non-alexithymic counterparts. Alexithymia is also associated with a higher relapse rate in alcohol dependent outpatients (Loas et al., 1997; Ziolkowski et al., 1995).

To date, there has been little research examining underlying factors or theoretical mechanisms of the relationship between alexithymia and alcohol dependence. A link between these factors is plausible based on an appraisal of relevant theories. Expectancy theory proposes that alcohol misuse is developed via mechanisms such as the positive and negative reinforcing effects of alcohol and vicarious learning (Bandura, 1977; Young and Oei, 1993). Drinking behavior is associated with outcome expectancies regarding the consequences of drinking, and these expectancies are reinforced by alcohol consumption (Miller et al., 1990; Young et al., 2006).

Alcohol expectancies include domains such as assertion, affective change and tension reduction (Young et al., 2006). Research has shown that individuals with positive alcohol expectancies drink higher quantities of alcohol and are more likely to misuse alcohol (Connor et al., 2000; Young and Oei, 1996). Affective change and tension reduction expectancies 
discriminate between individuals with alcohol dependence and controls (Young and Oei, 1996; Young et al., 2004). Only a single study has examined alexithymia and alcohol expectancies, reporting that alexithymia was associated with affect regulation expectancy and tension reduction expectancy (Junghanns et al., 2005).

Given that individuals with alexithymia often feel uncomfortable in social situations, alcohol may be used as a coping mechanism to enhance interpersonal functioning. Accordingly, we hypothesized that alcohol expectancies may be a plausible underlying mechanism of the relationship between alexithymia and alcohol dependence. The objective of the present study was to investigate alcohol expectancies related to emotional functioning as a mediator of this relationship.

\section{Methods}

\subsection{Participants}

Two hundred and thirty patients (158 males and 72 females) were recruited from a voluntary outpatient cognitive behavioral treatment program. The mean ages of the male and female participants were 36.82 years $(S D=11.38)$ and 38.77 years $(S D=9.18)$, respectively. Inclusion required a diagnosis of alcohol dependence (DSM-IV-TR, APA, 2000). Exclusion criteria included a co-morbid psychiatric disorder, substance dependence (other than nicotine), organic brain syndrome or heavy sedation.

\subsection{Measures}

2.2.1. Toronto Alexithymia Scale (TAS-20). The TAS-20 is a 20 item self-report measure of alexithymia (Bagby et al., 1994), with a higher score indicating higher levels of alexithymia. The TAS-20 has acceptable validity and reliability (Bagby et al., 1994; Parker et al., 2003; Thorberg et al., 2010). In this study, the Cronbach alpha was 0.85. 
2.2.2. Drinking Expectancy Questionnaire (DEQ). The DEQ is a 43 item self-report scale of alcohol expectancies that measures beliefs, emotions and thoughts associated with drinking. This study only examined three of the five primary factors-Assertion, Affective Change and Tension Reduction as these expectancies are specifically related to emotional functioning. The DEQ has sound psychometric properties (Young and Oei, 1996; Young et al., 2006). In this study, the Cronbach alphas for the Affective Change, Assertion and Tension Reduction scales were $0.84,0.78$ and 0.61 , respectively.

\subsubsection{Alcohol Use Disorders Identification Test (AUDIT). The AUDIT is a 10 item} instrument designed to measure alcohol problems (Babor et al., 2001). A total score of 20 or higher suggests dependent drinking. The AUDIT has sound psychometric properties (Reinert and Allen, 2007). In this study, the Cronbach alpha was 0.80.

\subsection{Procedure}

The study took place outside scheduled treatment sessions and instruments were administered before the first treatment session to reduce the confounding effects of therapy and medication. Participants were abstinent for a minimum of three days before assessment. Only patients not requiring detoxification were entered into the CBT program. Less than 3\% of the patients offered treatment declined.

\section{Results}

\subsection{Data Analysis Methods}

Descriptive statistics were performed for the TAS-20 total score, DEQ-Affective Change, DEQ-Assertion and DEQ-Tension Reduction scores and the AUDIT. Regression analyses were undertaken to establish the potential mediating roles of DEQ-Affective Change, 
DEQ-Assertion and DEQ-Tension Reduction on the relationship between TAS-20 total score and the AUDIT.

To test for mediation, a series of criteria must be met. The independent variable needs to be significantly correlated with the dependent variable and the independent variable has to be significantly correlated with the mediator. Furthermore, the mediator needs to be significantly related to the dependent variable even after controlling for the independent variable. If the independent variable is no longer significantly correlated with the dependent variable after controlling for the mediator, a perfect mediation is established. If only the first three criteria are met a partial mediation is established (Baron and Kenny, 1986).

\subsection{Descriptive statistics}

The mean score for the TAS-20 was $54.00(S D=12.16)$, DEQ-Affective Change $34.07(S D=9.25)$, DEQ-Assertion $37.43(S D=5.97)$, DEQ-Tension Reduction $15.51(S D=$ 2.97) and AUDIT $28.43(S D=6.79)$.

\subsection{Mediational Analyses}

Regression analyses were undertaken to examine the mediational effect of alcohol expectancies in the relationship between alexithymia and alcohol dependence severity. First, a single standard regression analysis showed a significant relationship between the predictor variable total, TAS-20, and the dependent variable, $\operatorname{AUDIT}, F(1,228)=12.14, p<.001$. TAS-20 scores accounted for $5.1 \%$ of the variance in AUDIT scores $\left(R^{2}=.051, p<.001\right)$. Second, a significant relationship was found between the predictor variable TAS-20 and the mediator DEQ-Affective Change, $F(1,228)=23.00, p<.0001$. The percentage of variance in TAS-20 scores accounted for by DEQ-Affective Change was 9.2\% $\left(R^{2}=.092, p<.0001\right)$. Third, a multiple regression analysis was performed with TAS-20 and DEQ-Affective Change 
to examine the mediational effect of the DEQ-Affective Change on the relationship between TAS-20 and AUDIT. The overall model was significant $F(2,227)=12.28, p<.0001$, and both TAS-20 $(\beta=.16, t(2)=2.36, p<.019)$ and DEQ-Affective Change $(\beta=.23, t(2)=3.44, p$ $<$.001) showed univariate significance (see Table 1).

To investigate if DEQ-Assertion mediated the relationship between the TAS-20 and AUDIT an identical set of regression analyses to those described above were conducted with the exception that instead of DEQ-Affective Change, DEQ-Assertion score was used. As shown in Table 1 there was a significant relationship between the predictor variable, TAS-20 and the dependent variable, AUDIT. A significant relationship was also found between the TAS-20 and the mediator DEQ-Assertion $F(1,228)=39.68, p<.0001$ accounting for $14.8 \%$ of the variance $\left(R^{2}=.148, p<.0001\right)$. Third, a multiple regression analysis was undertaken with TAS-20 and DEQ-Assertion to investigate the mediational effect of DEQ-Assertion on the relationship between TAS-20 and AUDIT, $F(2,228)=8.34, p<.0001$. The TAS-20 $(\beta=$ $.17, t(2)=2.44, p<.016)$ and DEQ-Assertion $(\beta=.15, t(2)=0.01, p=.038)$ yielded significant univariate effects (see Table 1). Given that the first three conditions of Baron and Kenny’s (1986) model were met for both mediators, but not the fourth criterion, these findings indicated that DEQ-Affective Change and DEQ-Assertion partially mediated the relationship between the TAS-20 and the AUDIT. As there was no significant relationship between TAS-20 and DEQ-Tension Reduction this expectancy was not examined as a potential mediator. Subsequent analyses ${ }^{1}$ were conducted on the TAS-20 subscales: Difficulties Identifying Feelings (DIF), Difficulties Describing Feelings (DDF) and Externally Oriented Thinking (EOT) following the same procedure as outlined above indicating that DEQ-Affective Change and DEQ-Assertion partially mediated the relationship between DIF

\footnotetext{
${ }^{1}$ Additional information about these analyses can be obtained from the corresponding author or on the Drug and Alcohol Dependence webpage under supplementary material.
} 
and AUDIT and fully mediated the relationship between DDF and AUDIT score. There was no significant relationship between EOT and AUDIT score.

\section{Discussion}

The present results suggested that the relationships between alexithymia and DIF with alcohol dependence severity were partially mediated through affective change and assertion expectancies. The relationship between DDF and alcohol dependence severity was fully mediated through these expectancy domains. Individuals with alexithymia and high affective change expectancy reported stronger emotions and increased negative emotional states when drinking. Thus, drinking did not appear to improve mood in those with alexithymia, but was associated with feelings of heightened negative mood. This may seem counterintuitive, however, previous evidence has shown that individuals with alexithymia are unable to differentiate somatic arousal and feelings leading to increased sympathetic activation (Infrasca, 1997; Neumann et al., 2004). The experience of an induced physiological state related to the depressant and mood altering effects of alcohol may in itself be reinforcing (Brick and Erickson, 1998; Winger et al., 2004).

An alternative explanation may be that alcohol facilitates the expression of the frustration related to alexithymia. A previous naturalistic study (Young and Oei, 2000) examining alcohol expectancies and alcohol use found that tension reduction was more related to "when' individuals drank than the effects obtained. Perhaps it is the cue state and not the actual outcome that is most relevant for the initiation of drinking in those with alexithymia and alcohol dependence. Affective change may be related to cues where improvement in mood is desired, but not necessarily achieved. Further research is needed to substantiate this hypothesis. 
The mediational relationships of assertion expectancies between alexithymia and alcohol dependence indicates that individuals with alexithymia report drinking alcohol in order to feel more outgoing, friendly and confident, as well as find it easier to express their feelings. There are some clear potential implications of this finding. For example, alcohol may help the person to "buffer" difficulties associated with the lack of emotional awareness in social settings. This may account for some of the interpersonal difficulties previously reported among those with substance use disorders (Thorberg and Lyvers, 2006).

Some caution should be exercised with these results. First, the cross-sectional design limits the interpretation of the findings and does not allow for the appraisal of affective change and assertion expectancies as causal mechanisms. Prospective research is needed to appraise causality. Second, the gender imbalance in a predominantly male sample potentially limits generalisability and studies comparing affective change and assertion as mechanisms across male and female alcohol dependent patients are needed. The present gender ratio is typical of alcohol dependent samples where there tends to be a larger number of males. Third, the use of self-report measures to assess key variables and response biases may have occurred. Future research should also use standardised measures to control for the effect of anxiety and depression on alexithymia.

The implications of our findings for treatment should be highlighted. Currently treatment interventions focus on helping patients alleviate 'bad' feelings, however, this approach may be less efficacious in individuals with alexithymia and alcohol dependence, as the present results suggest that this group drink alcohol to get access to stronger emotions. Thus, more specific assessment may be required in order to identify patients' with negative emotions related to their interpersonal experiences as well as an assessment of assertion. Given the need for replication of the current findings we suggest that therapeutic interventions 
focusing on a greater awareness of emotions that include an assertiveness component may be beneficial.

In conclusion, this study has provided empirical support for the importance of affective change expectancy and assertion expectancy as mechanisms partially explaining the relationship between alexithymia and alcohol dependence. The findings contribute to the theoretical integration of alexithymia with expectancy theory that may facilitate the development of improved assessment and treatment approaches for individuals with alexithymia and alcohol dependence. Future prospective research should investigate if interventions to develop skills to identify, describe and manage feelings, and increase assertiveness lead to decreased alcohol consumption and improved interpersonal functioning in individuals with alexithymia and alcohol dependence. 


\section{References}

American Psychiatric Association., 2000. Diagnostic and statistical manual of mental disorders (4th ed text rev. ed.). Washington, DC: American Psychiatric Association.

Babor, T. F., Higgins-Biddle, J. C., Saunders, J. B., Monteiro, M. G., 2001. Alcohol Use Disorders Identification Test: Guidelines for use in primary care (2nd ed.). Geneva: Switzerland: World Health Organization: Department of Mental Health and Substance Dependence.

Bagby, R. M., Parker, J. D. A., Taylor, G. J., 1994. The twenty-item Toronto Alexithymia Scale: I. Item selection and cross-validation of the factor structure. J Psychosom Res. 38, 23-32.

Bandura, A., 1977. Self-efficacy: Toward a unifying theory of behavioral change. Psychol Rev. 84, 191-215.

Baron, R. M., Kenny, D. A., 1986. The moderator-mediator variable distinction in social psychological research: Conceptual, strategic, and statistical considerations. J Pers Soc Psychol. 51, 1173-1182.

Brick, J., Erickson, C. K., 1998. Drugs, the brain, and behavior: The pharmacology of abuse and dependence. New York, NY: Haworth Press.

Connor, J. P., Young, R. M., Williams, R. J., Ricciardelli, L. A., 2000. Drinking restraint versus alcohol expectancies: Which is the better indicator of alcohol problems? J Stud Alcohol. 61, 352-359.

Infrasca, R., 1997. Alexithymia, neurovegetative arousal and neuroticism: An experimental study. Psychother Psychosom. 66, 276-280.

Junghanns, K., Tietz, U., Dibbelt, L., Kuether, M., Jurth, R., Ehrenthal, D. et al., 2005. Attenuated salivary cortisol secretion under cue exposure is associated with early relapse. Alcohol Alcohol. 40, 80-85. 
Loas, G., Fremaux, D., Otmani, O., Lecercle, C., Delahousse, J., 1997. Is alexithymia a negative factor for maintaining abstinence? A follow-up study. Comp Psych. 38, 296299.

Miller, P. M., Smith, G. T., Goldman, M. S., 1990. Emergence of alcohol expectancies in childhood: A possible critical period. J Stud Alcohol. 51, 343-349.

Nemiah, J. C., Freyberger, H., Sifneos, P. E., 1976. Alexithymia: a view of the psychosomatic process. In O. Hill (Ed.), Modern trends in psychosomatic medicine vol. 3. London: Butterworths.

Neumann, S. A., Sollers, J. J. I., Thayer, J. F., Waldstein, S. R. 2004. Alexithymia predicts attenuated autonomic reactivity, but prolonged recovery to anger recall in young women. Int J Psychophys. 53, 183-195.

Parker, J. D. A., Taylor, G. J., Bagby, R. M. 2003. The 20-item Toronto Alexithymia Scale III. Reliability and factorial validity in a community population. J Psychosom Res. 55, 269-275.

Reinert, D. F., Allen, J. P. 2007. The Alcohol Use Disorders Identification Test: An update of research findings. Alcohol Clin Exp Res. 31, 185-199.

Sakuraba, S., Kubo, M., Komoda, T., Yamana, J.I. 2005. Suicidal ideation and alexithymia in patients with alcoholism: A pilot study. Subst Use Misuse. 40, 823-830.

Thorberg, F. A., Lyvers, M. 2006. Attachment, fear of intimacy and differentiation of self among clients in substance disorder treatment facilities. Addict Behav. 31, 732-737.

Thorberg, F. A., Young, R. M., Sullivan, K. A., Lyvers, M. 2009. Alexithymia and alcohol use disorders: A critical review. Addict Behav. 34, 237-245.

Thorberg, F.A., Young, R.M., Sullivan, K.A., Lyvers, M., Connor., J. P., Feeney, G. F. X 2010. A psychometric comparison of the Toronto Alexithymia Scale (TAS-20) and 
the Observer Alexithymia Scale (OAS) in an alcohol-dependent sample. Personal Ind Differ. 43, 199-123.

Uzun, A. Z., Ates, A., Cansever, A., Ozsahin, A. 2003. Alexithymia in male alcoholics: Study in a Turkish sample. Comp Psych. 44, 349-352.

Winger, G., Woods, J. H., Hofmann, F. G. 2004. A handbook on drug and alcohol abuse: The biomedical aspects (4th ed.). New York, NY: Oxford University Press.

Young, R. M., Connor, J. P., Ricciardelli, L. A., Saunders, J. B. 2006. The role of alcohol expectancy and drinking refusal self-efficacy beliefs in university student drinking. Alcohol Alcohol. 41, 70-75.

Young, R. M., Oei, T. P. S. 1993. Grape expectations: The role of alcohol expectancies in the understanding of treatment of problem drinking. Int J Psychol. 28, 337-364.

Young, R. M., Oei, T. P. S. 1996. Drinking Expectancy Profile: Test Manual. Herston, Queensland, Australia: Department of Psychiatry, University of Queensland, Royal Brisbane Hospital.

Young, R. M., Oei, T. P. S. 2000. The predictive utility of drinking refusal self-efficacy and alcohol expectancy: A diary-based study of tension reduction. Addict Behav. 25, 415421.

Young, R. M. D., Lawford, B. R., Feeney, G. F., Ritchie, T., Noble, E. P. 2004. Alcoholrelated expectancies are associated with the D-2 dopamine receptor and GABA(A) receptor beta 3 subunit genes. Psych Res. 127, 171-183.

Young, R. M., Connor, J. P., et al. 2006. The role of alcohol expectancy and drinking refusal self-efficacy beliefs in university student drinking. Alcohol Alcohol. 41, 70-75.

Ziolkowski, M., Gruss, T., Rybakowski, J. K. 1995. Does alexithymia in male alcoholics constitute a negative factor for maintaining abstinence? Psychother Psychosom. 63, 169-173. 
Table 1.

DEQ-Affective change and DEQ-Assertion as mediators of the relationship between TAS-20 total scale and the AUDIT

\begin{tabular}{lllll}
\hline Variables & $R^{\Delta 2}$ & $B$ & $S E B$ & $\beta \mathrm{z}$ \\
\hline
\end{tabular}

AUDIT (DV)

Step 1

TAS-Total Scale

$\begin{array}{lll}.125 & .225\end{array}$

DEQ- Affective Change (DV)

Step 1

$.092 * *$

TAS-Total Scale

$\begin{array}{lll}.224 & .047 \quad 303\end{array}$

$\underline{\text { AUDIT (DV) }}$

Step 1

$.098 * *$

TAS-Total Scale

DEQ- Affective Change

$\begin{array}{lll}.087 & .037 & .156\end{array}$

$\begin{array}{lll}.172 & .050 & .228\end{array}$

DEQ- Assertion (DV)

Step 1

$.148^{* *}$

TAS-Total Scale

.189

.030

.385

AUDIT (DV)

Step 1

$.068 *$

TAS-Total Scale

.094

.039

.169

DEQ- Assertion

.165

.079

.145

$* P<.001 ; * * P<.0001$. AUDIT=Alcohol Use Identification Disorder Test, DEQ=Drinking Expectancy

Questionnaire, DV=Dependent Variable, TAS= Toronto Alexithymia Scale. 
Table 2. Means and SD for DIF, DDF and EOT

\begin{tabular}{ll}
\hline & Mean $(S D)$ \\
\hline DIF & $19.63(6.08)$ \\
DDF & $14.21(4.51)$ \\
EOT & $20.16(4.71)$ \\
\hline
\end{tabular}

DDF=Difficulties Describing Feelings, DIF=Difficulties Identifying Feelings, EOT=Externally Oriented Thinking. 
Table 3.

DEQ-Affective Change and DEQ-Assertion as mediators of the relationship between DIF and the AUDIT

\begin{tabular}{lllll}
\hline Variables & $R^{\Delta 2}$ & $B$ & $S E B$ & $\beta \mathrm{z}$ \\
\hline
\end{tabular}

$\underline{\text { AUDIT }}(\mathrm{DV})$

Step $1 \quad .083^{*}$

$\begin{array}{llll}\text { DIF } & .321 & .071 & .287\end{array}$

DEQ- Affective Change (DV)

Step 1

DIF

$.540 \quad .365$

$\underline{\text { AUDIT }}(\mathrm{DV})$

Step 1

$.116^{*}$

DIF

DEQ- Affective Change

$\begin{array}{lll}.241 & .075 & .216\end{array}$

$\begin{array}{lll}.148 & .051 & .196\end{array}$

DEQ- Assertion (DV)

Step 1

$.205 *$

DIF

$\begin{array}{lll}.444 & .058 & .453\end{array}$

AUDIT (DV)

Step 1

$.091 *$

DIF

.079

.242

DEQ- Assertion .114 .081 .101

$* P<.0001$. AUDIT=Alcohol Use Identification Disorder Test, DEQ=Drinking Expectancy Questionnaire, DIF= Difficulties Identifying Feelings, DV=Dependent Variable. 
Table 4.

DEQ-Affective Change and DEQ-Assertion as mediators of the relationship between DDF and the AUDIT

\begin{tabular}{lllll}
\hline Variables & $R^{\Delta 2}$ & $B$ & $S E B$ & $\beta \mathrm{z}$ \\
\hline
\end{tabular}

$\underline{\text { AUDIT }}(\mathrm{DV})$

Step $1+.017^{*}$

DDF

$.196 \quad .099 \quad .131$

DEQ- Affective Change (DV)

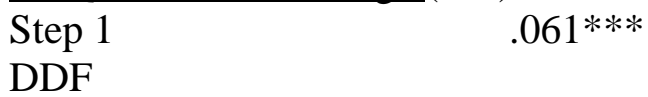

$\begin{array}{lll}.492 & .247\end{array}$

$\underline{\text { AUDIT }}(\mathrm{DV})$

\begin{tabular}{|c|c|c|c|}
\hline Step 1 & $.080 * * *$ & & \\
\hline DDF & .100 & .099 & .067 \\
\hline DEQ- Affective Change & .195 & .050 & .258 \\
\hline
\end{tabular}

DEQ- Assertion (DV)

Step 1

DDF

$.084^{* * *}$

$.384 \quad .084 \quad .291$

$\underline{\text { AUDIT }}(\mathrm{DV})$

Step 1

DDF

.114

.102

.076

DEQ- Assertion .214

.077

.188

$* P<.05 ; * * P<.01 ; * * * P<.0001$. AUDIT=Alcohol Use Identification Disorder Test, DEQ=Drinking

Expectancy Questionnaire, DDF=Difficulties Describing Feelings, DV=Dependent Variable. 